\title{
Differential Outgrowth of Axons and their Branches Is Regulated by Localized Calcium Transients
}

\author{
B. Ian Hutchins ${ }^{2}$ and Katherine Kalil ${ }^{1,2}$ \\ ${ }^{1}$ Department of Anatomy and ${ }^{2}$ Neuroscience Training Program, University of Wisconsin-Madison, Madison, Wisconsin 53706
}

\begin{abstract}
During development axon outgrowth and branching are independently regulated such that axons can stall or retract while their interstitial branches extend toward targets. Previous studies have shown that guidance cues and intracellular signaling components can promote branching of cortical axons without affecting axon outgrowth. However, the mechanisms that regulate differential outgrowth of axons and their branches are not well understood. Based on our previous work showing the importance of localized repetitive calcium transients in netrin-1-induced cortical axon branching, we sought to investigate the role of calcium signaling in regulating differential outgrowth of axons and their branches. Using fluorescence calcium imaging of dissociated developing cortical neurons, we show that localized spontaneous calcium transients of different frequencies occur in restricted regions of axons and their branches. Higher frequencies occur in more rapidly extending processes whereas lower frequencies occur in processes that stall or retract. Direct induction of localized calcium transients with photolysis of caged calcium induced rapid outgrowth of axonal processes. Surprisingly outgrowth of one axonal process was almost invariably accompanied by simultaneous retraction of another process belonging to the same axon, suggesting a competitive mechanism for differential process outgrowth. Conversely, reducing frequencies of calcium transients with nifedipine and TTX reduced the incidence of differential process outgrowth. Together these results suggest a novel activity-dependent mechanism whereby intrinsic localized calcium transients regulate the competitive growth of axons and their branches. These mechanisms may also be important for the development of cortical connectivity in vivo.
\end{abstract}

Key words: calcium signaling; axon outgrowth; axon branching; cortical development; calcium imaging; neural activity

\section{Introduction}

In vivo (Bastmeyer and O'Leary, 1996; Portera-Cailliau et al., 2005; Meyer and Smith, 2006) and in vitro (Ruthel and Hollenbeck, 2000; Dent et al., 2004), the outgrowth of axons and the extension of branches are independently regulated such that branches can extend while their axons stall or retract (Luo and O'Leary, 2005). However, the mechanisms whereby axons and their branches can have differential rates of outgrowth are unknown. Calcium has been shown to have varying effects on axon outgrowth and guidance (Henley and Poo, 2004; Gomez and Zheng, 2006). In most cases, calcium activity was measured in the axon or the growth cone and levels of intracellular $\mathrm{Ca}^{2+}$ were found to be inversely correlated with rates of axon outgrowth (Gomez and Spitzer, 1999; Tang et al., 2003; Jacques-Fricke et al., 2006) or changes in the direction of axon growth (Nishiyama et al., 2003; Henley et al., 2004; Wen et al., 2004). In previous studies, we found that global $\mathrm{Ca}^{2+}$ transients inhibited extension of the primary axon (Tang et al., 2003), whereas localized $\mathrm{Ca}^{2+}$ transients evoked by netrin-1 signaling could promote branching

Received May 29, 2007; revised 0ct. 31, 2007; accepted Nov. 1, 2007.

This work was supported by National Institutes of Health (NIH) Grant NS14428 and by a grant from the Whitehall Foundation. B.I.H. was supported by an Advanced Opportunity Fellowship, Predoctoral Training Grant GM07075, and a Research Supplement for Underrepresented Minorities from the NIH. We thank Dr. Timothy Gomez for help with the photolysis experiments and helpful advice during the course of this work.

Correspondence should be addressed to Katherine Kalil, Department of Anatomy, University of WisconsinMadison, 1300 University Avenue, Madison, WI 53706. E-mail: kakali@@facstaff.wisc.edu.

DOI:10.1523/JNEUROSCI.4548-07.2008

Copyright $\odot 2008$ Society for Neuroscience $\quad$ 0270-6474/08/280143-11\$15.00/0 of the axon in regions of high-frequency $\mathrm{Ca}^{2+}$ transients (Tang and Kalil, 2005). It has been well established that competitive activity-dependent mechanisms can regulate synaptic innervation at target sites. The competition among neighboring axon arbors for synaptic space was shown previously to favor arbors with higher levels of neural activity (Hua and Smith, 2005). Because electrical activity is reflected by changes in intracellular $\mathrm{Ca}^{2+}$ (Spitzer, 2006), we hypothesized that differences in $\mathrm{Ca}^{2+}$ levels could also be a mechanism for regulating the differential growth of axons and their branches.

However, competitive mechanisms for growth of axons and collateral branches of the same axon have been little studied. Previous studies of trophic factor-mediated axon growth (Goldberg et al., 2002; Singh and Miller, 2005) showed that electrical activity enhanced axon outgrowth. In populations of sympathetic neurons grown in divided chambers axons depolarized experimentally with $\mathrm{KCl}$ in one chamber extended longer axons at the expense of their unstimulated branches in another chamber (Singh and Miller, 2005). However, it was not known whether a developing neuron could spontaneously exhibit different levels of activity in axon branches belonging to the same axon. To address this question, we asked whether dissociated cortical neurons could express differential $\mathrm{Ca}^{2+}$ activity in axons versus branches. Imaging $\mathrm{Ca}^{2+}$ activity in isolated neurons permitted us to obtain accurate measurements of frequencies of $\mathrm{Ca}^{2+}$ activity. Importantly we were able to correlate differences in frequencies of $\mathrm{Ca}^{2+}$ transients with differential outgrowth of axons and their 
branches. A further advantage of this approach was the ability to impose $\mathrm{Ca}^{2+}$ transients of specific frequencies with photolysis of caged calcium to restricted regions of axons and branches. Here we show that differential frequencies of $\mathrm{Ca}^{2+}$ transients in a cortical axon and its branch favor the growth of the process with higherfrequency transients while at the same time the process with lower frequency transients is actively retracted. These results suggest a competitive mechanism whereby higherfrequency $\mathrm{Ca}^{2+}$ activity in one axonal process promotes its extension at the expense of another process of lower $\mathrm{Ca}^{2+}$ activity that stalls or retracts.

\section{Materials and Methods}

Cell culture. Primary cortical neurons were prepared from the sensorimotor cortex of postnatal day 0 (P0)-P3 Syrian hamster pups as described previously (Dent and Kalil, 2003). Primary neurons were dissociated and plated onto acidetched coverslips coated with $0.5 \mathrm{mg} / \mathrm{ml}$ poly-Dlysine (Sigma, St. Louis, MO) or $2.5 \mu \mathrm{g} / \mathrm{cm}^{2}$ fibronectin (Sigma; photolysis experiments only) at a density of $1-$ to $3-\mathrm{k}$ cells $/ \mathrm{cm}^{2}$. Cultures were then incubated in $5 \% \mathrm{CO}_{2}$ and $9 \% \mathrm{O}_{2}$ at $37^{\circ} \mathrm{C}$ for $24-48 \mathrm{~h}$ until imaging.

Experimental reagents. Stock solutions were prepared by dissolving drugs in water or dimethyl sulfoxide (DMSO) according to the recommendations of the manufacturer. Stock solutions were then diluted into serum-free medium and bath applied to cultures, with the exception of netrin-1, which was locally applied. The following drugs were used to modulate calcium activity: L-type voltage-gated calcium channel inhibitor nifedipine (10 $\mu \mathrm{m}$; Calbiochem, La Jolla, CA) and activator BayK 8644 (BayK; $20 \mu \mathrm{M}$; Alomone Labs, Jerusalem, Israel), recombinant chicken protein netrin-1 ( $25 \mu \mathrm{g} / \mathrm{ml}$; R\&D Systems, Minneapolis, $\mathrm{MN})$, voltage-gated sodium channel inhibitor tetrodotoxin (TTX; $1 \mu \mathrm{M}$; Calbiochem), and caged calcium $o$-nitrophenyl (NP)-EGTA AM ( $4 \mu \mathrm{M}$; Invitrogen, Eugene, OR).

Measurements of calcium activity. Calcium imaging and analysis were performed as previously described (Tang et al., 2003; Tang and Kalil, 2005). Briefly, 18-72 $\mathrm{h}$ after plating cell cultures were loaded with $4 \mu \mathrm{M}$ Fluo4 AM (Invitrogen) for $30 \mathrm{~min}$. After loading, the medium was changed and cultures were allowed to recover for $30 \mathrm{~min}$ in the incubator before imaging. Under these conditions, Fluo-4 brightly labeled every cell examined. Fluorescence time-lapse images were taken every 5-10 s with a Princeton Instruments (Trenton, NJ) MicroMax 512 BFT cooled CCD camera mounted on an inverted Nikon (Tokyo, Japan) TE300 epifluorescence microscope while cultures were maintained at $37^{\circ} \mathrm{C}$ with an incubator blower. Images were acquired and binned under low light conditions, increasing the sensitivity of the camera while maintaining low levels of background noise. Image acquisition and analysis were performed with MetaMorph image processing software (Molecular Devices, Union City, CA).

Calcium activity was measured as the average fluorescence pixel intensity $(F)$ in an axon region divided by the baseline fluorescence in that region $(F 0)$. Background fluorescence was measured frame-by-frame in an empty region of the viewing field and was subtracted from measure-
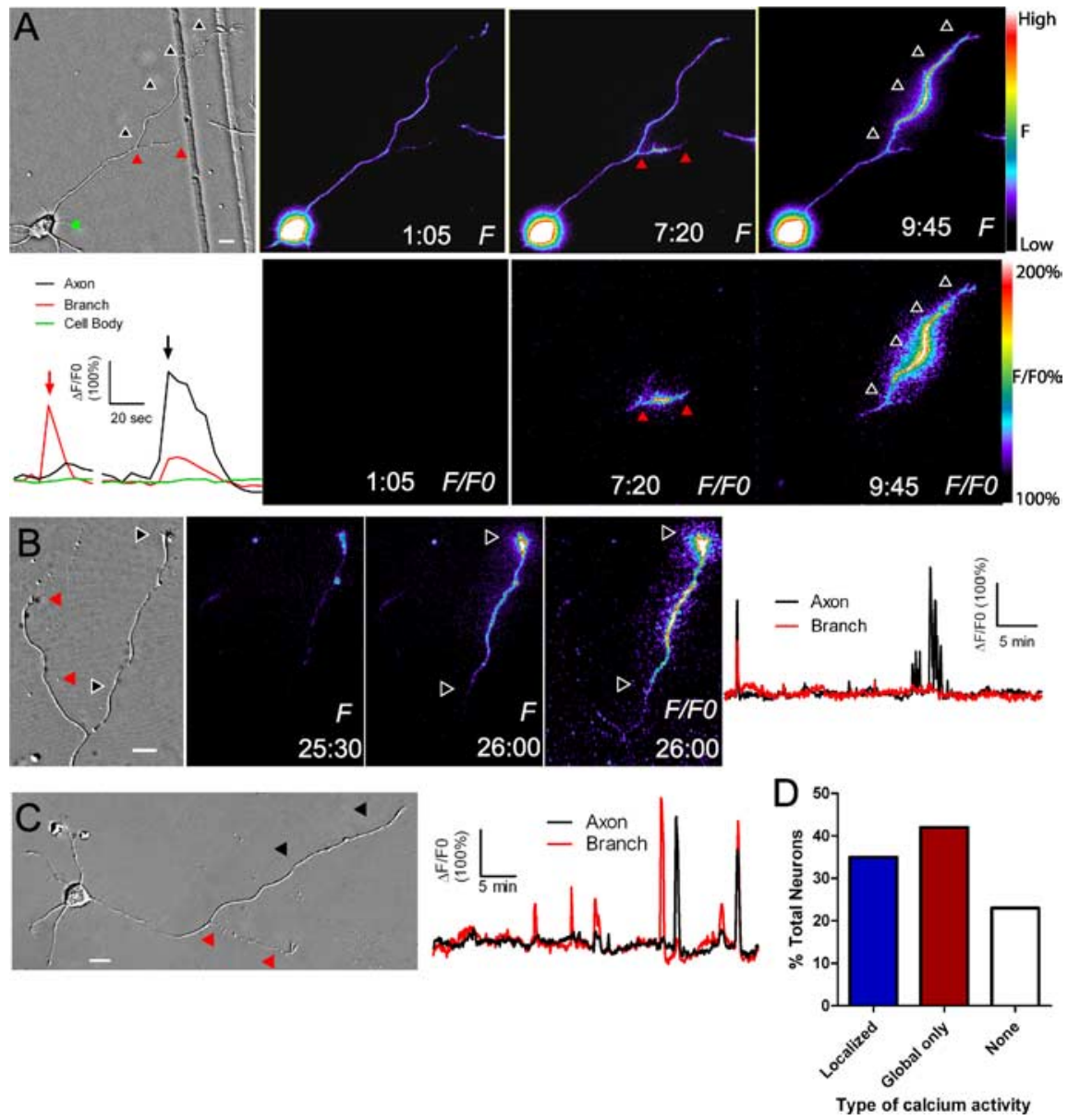

Figure 1. Localized calcium transients occur in restricted regions of primary axons or their collateral branches. $\boldsymbol{A}, \mathrm{DIC}$ image (top left) and fluorescence images of calcium activity (panels labeled $F$ ) in a cortical neuron in which calcium transients are localized to the primary axon (time point 9:45) or its collateral branch (time point 7:20). At 1:05, the neuron shows no calcium activity. Arrowheads (black and red) correspond to regions of localized calcium activity. Panels labeled F/FO correspond to the ( (he primary axon (black), branch (red) and cell body (green) during the calcium transients shown in the indicated by black arrowheads in the primary axon of a cortical neuron (right panels). As shown in the graph of calcium activity (far right), localized calcium transients occurred only in the primary axon (black), making its average frequency of calcium transients much higher than that of the branch (red). One global calcium transient occurred near the beginning of the imaging session. Calibration bars as in $\boldsymbol{A}$. C, Both the primary axon (black arrowheads) and branch (red arrowheads) show localized neurons with different types of calcium activity. Pseudocolor scales are the same for all images of raw fluorescence and normalized fluorescence, respectively. Times are given in minutes and seconds. Scale bars, $10 \mu \mathrm{m}$.

ments of fluorescence intensity. To minimize the effects of any morphological changes that could affect fluorescence measurements through changes in volume, the baseline ( F0) was calculated as a shifting average of the fluorescence intensity over a 5-min window. Calcium transients were defined as fluorescence signals $(F / F 0)$ that exceed $150 \%$ of the baseline fluorescence, which were confirmed by frame-by-frame analysis of the time-lapse images. To ensure that random fluctuations were not counted as calcium transients we included a requirement that the peak amplitude of transients be at least 2 SDs from baseline. To measure the spatial extent of localized calcium transients, the background-subtracted fluorescence image $(F)$ was divided by an average of the frames preceding the localized calcium event $(F 0)$ and borders were defined as the points where the signal $(F / F 0)$ dropped to $110 \%$. If the signal did not drop to $110 \%$ before reaching a branch point the localized calcium transient was considered to encompass the entire branch and was not included in the analysis of length of localized calcium transients. Calcium signals with no localization to an axonal process or region of an axonal process were 
A
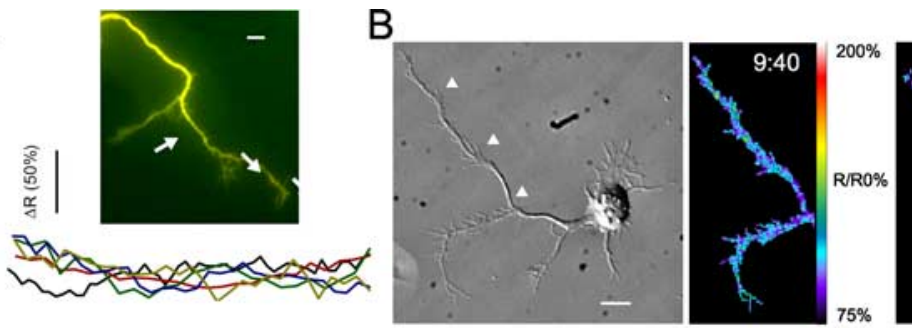

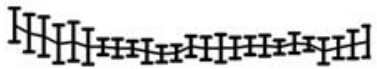

C
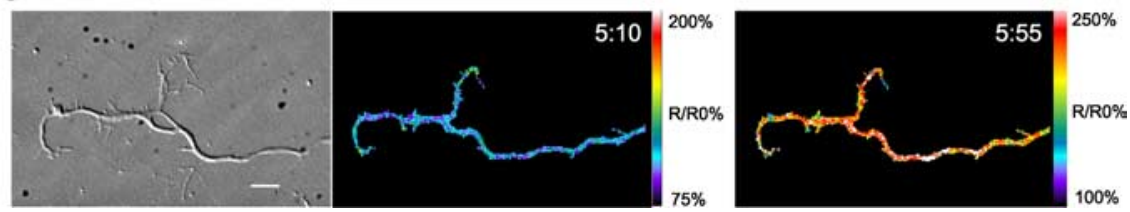

Figure 2. Ratiometric imaging controls for differences in cell volume over time and reveals that amplitudes of global calcium transients are larger than those of localized calcium transients. $\boldsymbol{A}$, Overlay of Fluo-4 (green) and volumetric marker Calcein Red/Orange (red) fluorescence shows uniform loading of both dyes (top). Arrows indicate regions through the axon from which line scans were obtained. Linescans through axonal processes of five neurons (shown below in different colors) during 5 min periods when calcium activity did not change showed that the ratio of fluorescence intensities over the entire axonal process remained constant. $\boldsymbol{B}, \mathrm{DIC}$ image (left) and corresponding ratiometric images of calcium activity $(R / R 0$, middle, right) of a cortical neuron at rest (middle, 9:40) and later exhibiting a localized calcium transient (right, 10:00). Arrowheads indicate corresponding positions on DIC and fluorescence images. The pseudocolor calibration bars are shown at right. C, DIC image (left) and ratiometric images of calcium activity $(R / R 0$, right) of a cortical neuron at rest (middle, 5:10) and later exhibiting a global calcium transient (right, 5:55). Scale bars, $10 \mu \mathrm{m}$.

considered to be global, although it was not always possible to image the entire neuron in the same field of view to confirm the global nature of the calcium transient.

For ratiometric experiments, cultures were loaded with $4 \mu \mathrm{M}$ Fluo4 $\mathrm{AM}$ and $250 \mathrm{~nm}$ volumetric indicator Calcein Red/Orange (Invitrogen) for $30 \mathrm{~min}$. Fluorescence images of Calcein Red/Orange were acquired immediately after each frame of Fluo-4 fluorescence. Ratiometric measurements $(R)$ were obtained by dividing the Fluo-4 fluorescence value by the fluorescence value of Calcein Red/Orange. Frame-by-frame background subtraction was performed for each indicator as described above. Calcium signals $(R / R 0)$ were then measured as the percentage change from a shifting average baseline $(R 0)$ of the ratiometric measurements as described above for nonratiometric measurements.

After imaging, the frequency and amplitude of calcium transients in a region of each axon process were measured and analyzed with computer algorithms in Microsoft (Redmond, WA) Excel. Regions in each axonal process were chosen around the areas of maximal calcium activity and did not change throughout the imaging session. Relative frequencies of calcium transients expressed in two branches of the same axon were calculated as the frequency of calcium transients in the process of interest divided by the sum of the frequencies of calcium transients in each process. This ratio of calcium activity was then correlated with the rate of outgrowth for each process.

Measurements of rates of process outgrowth. Outgrowth rates were measured as the difference in axon or branch length between differential interference contrast (DIC) or phase-contrast images acquired at the beginning and end of the imaging session, divided by the duration of the imaging session. For short term experiments DIC images were acquired immediately before and after calcium imaging. For long term BayK 8644 and silencing experiments phase contrast images were acquired on a Zeiss (Thornwood, NY) Axiovert 35M inverted microscope with a $20 \times$ objective. Drugs were applied after $24-48 \mathrm{~h}$ in vitro, and axon and branch length were recorded at the beginning and end of the drug treatment, which lasted up to $24 \mathrm{~h}$. For quantification of differential outgrowth in BayK and silencing experiments, differences in outgrowth rates of axons and their branches were considered significant if the magnitude of the difference in outgrowth rates was at least twice the average rate of out-

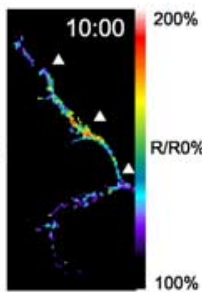

growth for that experiment. Only isolated neurons, that had a branched axon at least twice as long as the next longest primary neurite, were selected for imaging and analysis. Processes longer than $20 \mu \mathrm{m}$ extending from the axon were considered branches.

Local application of netrin-1. Local application of netrin-1 was performed as described previously (Tang and Kalil, 2005). Briefly, pulled pipettes with $0.5-1 \mu \mathrm{m}$ tips connected to an Eppendorf (Hamburg, Germany) microinjection system were loaded with $25 \mu \mathrm{g} / \mathrm{ml}$ stock solution of netrin-1 and positioned nearby (10-20 $\mu \mathrm{m})$ axons or branches. Netrin was puffed out of the pipettes by a pressure of $20-40 \mathrm{kPa}$ every second for imaging periods up to an hour. Calcium activity was measured during local application of netrin-1 and outgrowth of existing and de novo branches was measured from DIC images as described above.

Caged calcium experiments. Neurons were loaded with $4 \mu \mathrm{M}$ NP-EGTA AM for $30 \mathrm{~min}$, then rinsed and allowed to recover for $30 \mathrm{~min}$ before imaging. Caged calcium was released by photolysis of NP-EGTA with $360 \mathrm{~nm}$ UV light from a $100 \mathrm{~W}$ mercury lamp filtered to $25 \%$ intensity with a neutral density filter. The size of the exposed region was restricted to a column of light up to $50 \mu \mathrm{m}$ in diameter by placing a pinhole aperture in the light path. A programmable Uniblitz (Rochester, NY) shutter controlled opening and closing of the shutter, exposing a region of the axon up to $50 \mu \mathrm{m}$ in diameter to a UV light pulse $200 \mathrm{~ms}$ in duration. To confirm that photolysis of NP-EGTA increased calcium activity some cultures were coloaded with NP-EGTA and Fluo-4, and calcium activity was measured concurrently with photolysis on an Olympus (Center Valley, PA) Fluoview 500 laser-scanning confocal system mounted on an AX-70 upright microscope with a $40 \times$ fluorite objective. Outgrowth was measured from DIC images as described above. Control neurons were exposed to UV light without loading with NP-EGTA. To acquire higher-resolution DIC images, additional experiments were performed on the inverted Nikon TE300 epifluorescence system. No significant differences in response to photolysis of caged calcium were observed between these two systems. However, because simultaneous calcium imaging during photolysis to measure the size of evoked calcium transients could not be performed on the Nikon system, these additional experiments were omitted from the quantifications of outgrowth.

Statistical analysis and image processing. Graphs and statistical analysis were performed with GraphPad (San Diego, CA) Prism statistical analysis software. Unless otherwise noted, comparisons between two groups were made with Student's $t$ test and comparisons between multiple groups were made with a one-way ANOVA with Tukey's posttest. Measurements are given in mean \pm SEM unless otherwise noted. The DIC images presented in figures were enhanced with a high-pass filter and brightness-contrast adjustments in Adobe (Mountain View, CA) Photoshop. Images representing raw fluorescence data $(F)$ were not modified except to perform background subtraction with an image of background fluorescence taken from an empty field of view. Images representing the change in calcium signal ( $F / F 0$ and $R / R 0$, as described above) were additionally passed through a $2 \times 2$ low-pass filter in MetaMorph to reduce single-pixel noise. Supplemental time-lapse images (available at www.jneurosci.org as supplemental material) were assembled into Apple $(\mathrm{Cu}-$ pertino, CA) QuickTime movies with Adobe Premiere software.

\section{Results}

Localized $\mathrm{Ca}^{2+}$ transients of different frequencies can occur in axons and their branches

Cortical neurons exhibit calcium transients that are either global or localized (Tang et al., 2003; Tang and Kalil, 2005). Previously, 
we found that global transients were characterized by spontaneous fluctuations in levels of $\mathrm{Ca}^{2+}$ that appeared instantaneously throughout the entire neuron and occurred at frequencies averaging $\sim 1$ transient per minute. In the present study we found spontaneous localized $\mathrm{Ca}^{2+}$ transients that were typically confined to a region of the axon or to a branch or were expressed alternately in the primary axon and a branch (Fig. 1). Here we were able to measure localized $\mathrm{Ca}^{2+}$ transients confined to regions of the axon or a branch as small as $20 \mu \mathrm{m}$ in length (Fig. $1 A, B$ ). To define the borders of the localized calcium transients we adopted the criterion that the amplitude of the localized calcium signal was $110 \%$ of the baseline fluorescence (see Materials and Methods). Localized regions of calcium activity in the axon or branch averaged $45.8 \pm 4 \mu \mathrm{m}$ (mean \pm SEM, $n=$ 24 ), a measurement that excluded localized transients encompassing an entire branch.

Measurements of the amplitude of $\mathrm{Ca}^{2+}$ transients based on comparisons between the signal and the baseline $(F / F 0)$ are accurate as long as the axonal process does not change shape during the imaging session. Nevertheless, we decided to obtain a more accurate characterization of the amplitudes of localized axonal transients by performing ratiometric imaging with a volumetric marker. As shown in the example in Figure $2 B$, the axonal branch (white arrowheads) has a region of high $\mathrm{Ca}^{2+}$ activity whose peak amplitude is $>200 \%$ of baseline, which takes any volumetric changes into account. Importantly, line scans through the axonal areas of interest in five different neurons show that the concentration of Fluo-4 is uniform (Fig. 2A), demonstrating that our measurements of amplitudes of $\mathrm{Ca}^{2+}$ activity are not affected by an uneven distribution of the indicator dye. The area of localized $\mathrm{Ca}^{2+}$ activity $(48 \mu \mathrm{m})$ is within the average axon length calculated by nonratiometric imaging. These ratiometric measurements therefore validate our criteria for characterizing localized $\mathrm{Ca}^{2+}$ transients and also made it possible to compare the amplitudes of localized and global in different neurons (Fig. $2 \mathrm{~B}, \mathrm{C}$ ). Global $\mathrm{Ca}^{2+}$ transients had an average amplitude of $231 \pm 4 \%$ of baseline whereas amplitudes of localized $\mathrm{Ca}^{2+}$ transients averaged $175 \pm 8 \%$ of baseline $(p<$ $0.001, t$ test; $n \geq 6$ independent experiments for each condition). It is clear that localized regions do not correspond to microdomains involving the cytoplasm in close proximity to small clusters of calcium channels (Augustine et al., 2003). Measurements of $\mathrm{Ca}^{2+}$ activity in 81 cortical neurons showed that $77 \%$ exhibited spontaneous transients. Of these $45 \%$ showed localized $\mathrm{Ca}^{2+}$ transients and of these $55 \%$ exhibited both local and global transients (Fig. 1D). The remaining 55\% of neurons had only global transients.

As we had noted previously (Tang and Kalil, 2005) netrin-1 $10 \mu \mathrm{m}$.
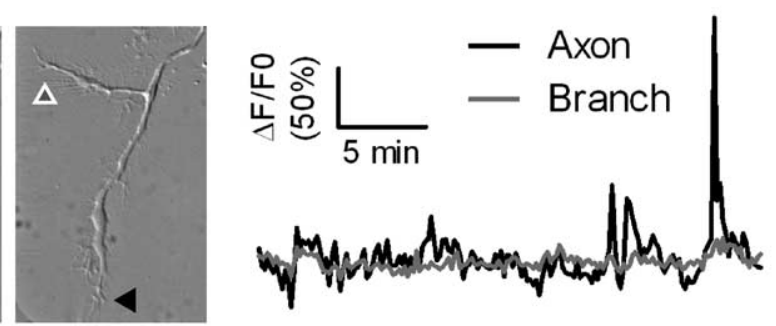

C

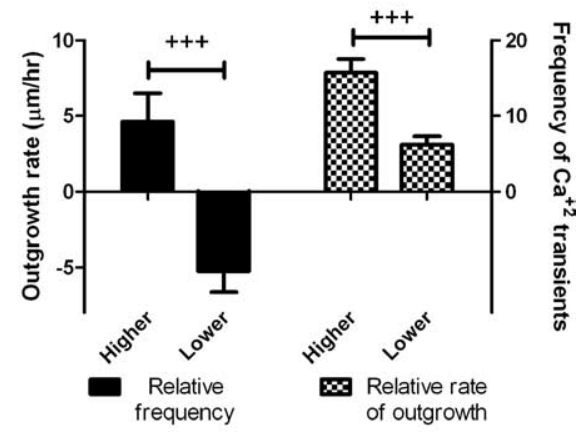

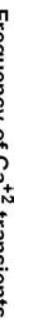

Figure 3. Differential calcium activity correlates with differential outgrowth of cortical axons and their branches. $\boldsymbol{A}, \mathrm{DIC}$ images of an axon (black arrowhead) and its branch (white/gray arrowhead) with different levels of spontaneous calcium activity transients and grew only $1.2 \mu \mathrm{m}$. B, Frequencies of calcium transients in individual axonal processes do not correlate absolutely with rates of outgrowth ( $p>0.05$, one-way ANOVA). $\boldsymbol{C}, \boldsymbol{D}$, Direct comparisons were made among processes from the same axon rocesses with higher frequencies of calcium transients had higher rates of outgrowth, whereas processes with lower frequencies outgrowth in single axonal processes to their relative frequencies of calcium transients $(n=56)$. Relative frequencies were calculated as the ratio of the frequency in the process of interest to the frequency in another process from that axon, expressed as a percentage. Processes with the highest relative frequencies had the highest rates of outgrowth whereas processes with the lowest relative frequencies had the highest rates of retraction ( ${ }^{*} p<0.05$, ANOVA on ranks with Dunn's post-test). Scale bar,

evoked $\mathrm{Ca}^{2+}$ transients alternating between two axonal processes could have different frequencies. Because spontaneous $\mathrm{Ca}^{2+}$ transients occurred in localized regions of axons and their branches we asked whether these transients also exhibited different frequencies. We found that calcium transients were not only localized but that in most of these cases neurons exhibited $\mathrm{Ca}^{2+}$ transients of different frequencies in a region of an axon vs a branch or in one branch vs another branch of the same axon. $\mathrm{Ca}^{2+}$ transients were defined as events that exceeded $150 \%$ of a shifting average baseline of fluorescence intensity (see Materials and Methods). To distinguish real events from random fluctuations, we also required that the events be 2 SDs from baseline. Of 20 neurons showing spontaneous differential $\mathrm{Ca}^{2+}$ activity in their axons and branches, frequencies ranged from 1.5 to $30 / \mathrm{h}$ and averaged 11.1 transients/h. Measurements of frequencies of repetitive localized $\mathrm{Ca}^{2+}$ transients were all made in the same 

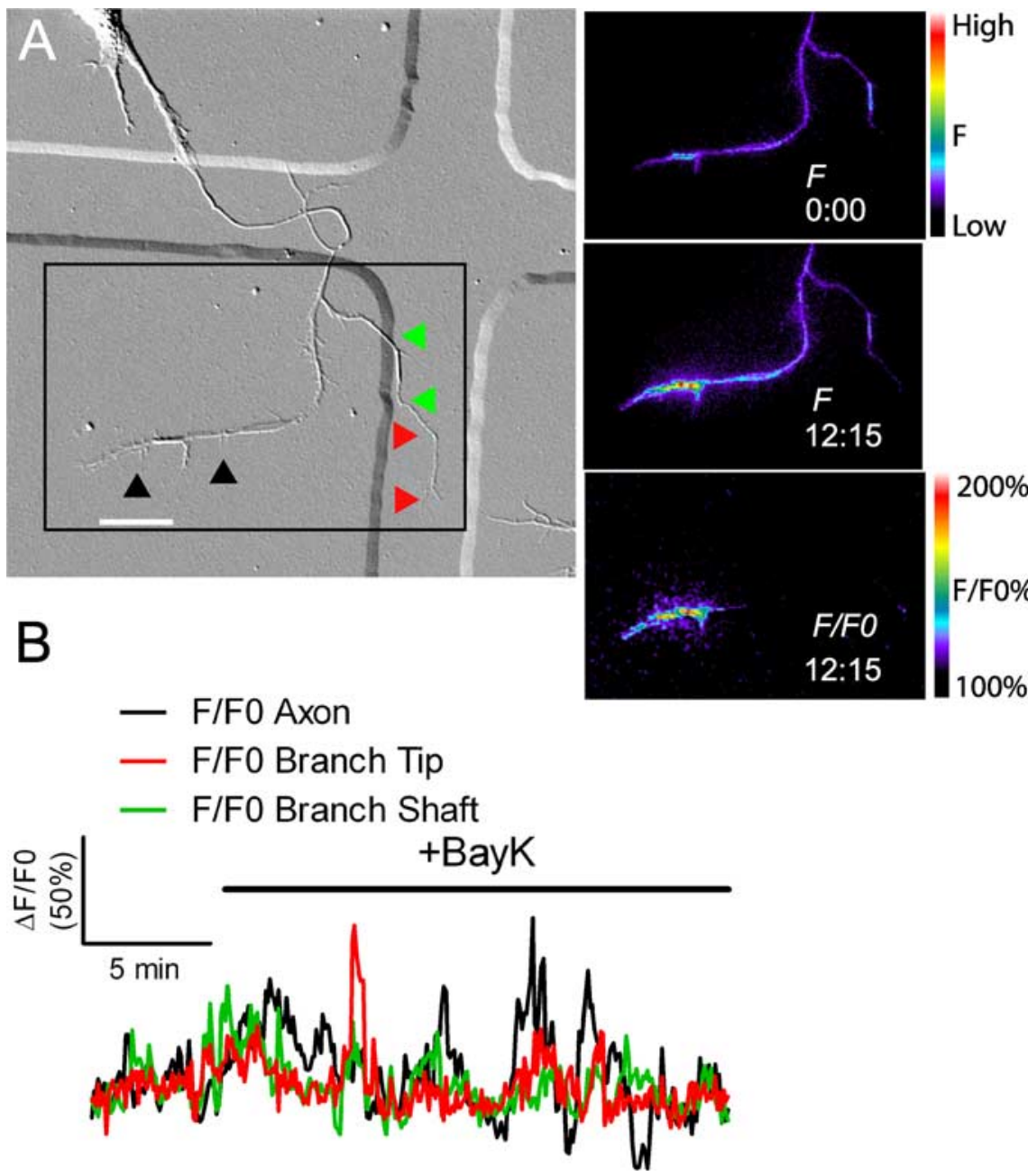

Figure 4. Bath application of BayK 8644 induces localized calcium transients. A, DIC (left) and fluorescence images (right) of a cortical neuron exposed to BayK 8644. Bath application of BayK at 0:00 induced high-frequency localized calcium transients primarily in the axon (between black arrowheads), but also in the branch (red and green arrowheads). An example of a localized calcium transient evoked by BayK is shown at time point 12:15. Calibration bars for raw fluorescence $(F)$ and normalized fluorescence $(F / F O)$ are shown at right. $B$, Graph of calcium activity before and after application of BayK (indicated by the black bar). Colors in the graph correspond to arrowheads in $A$. BayK 8644 induced localized calcium activity in six of seven experiments. Scale bar, $20 \mu \mathrm{m}$.

spatial extent of the axon which did not significantly change over time. As shown in Figure 1, $B$ and $C$, frequencies of localized $\mathrm{Ca}^{2+}$ transients in both neurons differed in their two axonal processes. In the example in Figure $1 B$, only the process at right (black arrow) showed localized activity whereas the branch at left (red arrow) had no measurable activity (see also supplemental Movie 1, available at www.jneurosci.org as supplemental material). Note that at the beginning of the image sequence a large global $\mathrm{Ca}^{2+}$ transient occurred. In Figure $1 C$, both the axon and its branch had localized transients which were of different frequencies (6.3 vs 2.1 transients/h).

\section{Differential $\mathrm{Ca}^{2+}$ activity is correlated with differential outgrowth of axons and their branches}

To determine whether frequencies of $\mathrm{Ca}^{2+}$ transients were correlated with rates of process outgrowth, we first measured the frequencies of $\mathrm{Ca}^{2+}$ transients in axons with at least one branch that was at least $20 \mu \mathrm{m}$ in length. Figure $3 A$ shows an example of an axon and a branch each with spontaneous localized $\mathrm{Ca}^{2+}$ transients of a different frequency. During the $30 \mathrm{~min}$ imaging period the axon had 8.37 transients/h localized to the region of the growth cone whereas the branch had no localized activity. During the imaging period, there were no global transients. We then compared DIC images taken at the beginning and end of the imaging session to calculate the rate of process outgrowth. The axon tip had advanced $14 \mu \mathrm{m}(28 \mu \mathrm{m} / \mathrm{h})$, but the branch grew only $1.2 \mu \mathrm{m}$ during this time period. These results seem to suggest that higherfrequency $\mathrm{Ca}^{2+}$ transients promote process outgrowth. However, additional analysis revealed that the relationship between frequency of $\mathrm{Ca}^{2+}$ transients and process outgrowth was not simply linear (Fig. 3B). Instead, differences in rates of outgrowth between processes of the same axon were dependent on relative differences in their frequencies of $\mathrm{Ca}^{2+}$ transients. In 20 neurons in which localized $\mathrm{Ca}^{2+}$ transients were observed in axons and their branches, we found that, in most cases, the processes with higher-frequency transients were favored for growth (Fig. 3C). In many cases (76\%) (Fig. 3C,D) processes with lowerfrequency $\mathrm{Ca}^{2+}$ transients actually retracted, suggesting a competitive effect in which the growth of the favored process occurred at the expense of another with lower frequency $\mathrm{Ca}^{2+}$ transients. Moreover, the greatest differences in outgrowth of two different processes of the same axon occurred when differences in frequencies were the highest (Fig. 3D). As shown in Figure $3 D$, the differences in relative frequency of $\mathrm{Ca}^{2+}$ transients in two axonal processes were proportional to the differences in their rates of outgrowth. Higher growth rates of axonal processes were correlated with higher frequencies of $\mathrm{Ca}^{2+}$ transients regardless of whether the process was an axon or a branch (Fig. 3). Moreover these transients could occur along the shaft or at the growth cone of the axonal process.

Because global $\mathrm{Ca}^{2+}$ transients often occurred during the same sequences in which localized transients were measured, it was important to determine whether global transients also contributed to differential process outgrowth. In neurons that exhibited only global transients $(n=20)$, differences in rates of growth for two processes of the same axon were relatively small compared with differences in rates of growth for processes that had localized $\mathrm{Ca}^{2+}$ transients of different frequencies $(4.8 \pm 1.4$ $\mu \mathrm{m} / \mathrm{h}$ vs $10.9 \pm 2 \mu \mathrm{m} / \mathrm{h} ; p<0.005$, rank sum test). These data show that global transients contribute relatively little to differential process outgrowth.

\section{Localized $\mathrm{Ca}^{2+}$ transients evoked with BayK and netrin-1} promote differential outgrowth of axons and their branches All of our measurements thus far involved endogenous $\mathrm{Ca}^{2+}$ transients. To induce localized $\mathrm{Ca}^{2+}$ transients, we bath applied BayK 8644, which has been shown to open L-type voltage-gated $\mathrm{Ca}^{2+}$ channels. In most cases in which $\mathrm{Ca}^{2+}$ activity was imaged, 
BayK evoked an initial burst of global activity followed by localized $\mathrm{Ca}^{2+}$ activity ( 6 of 7 experiments) but in one case only global activity occurred. As shown in Figure $4 A$, application of BayK to neurons showing little fluctuation in $\mathrm{Ca}^{2+}$ activity almost immediately evoked localized highfrequency transients in the axon (black arrowhead) and asynchronous transients of lower-frequency (Fig. 4B) in the branch (red arrowhead). These transients persisted throughout the imaging period (see also supplemental Movie 2, available at www.jneurosci.org as supplemental material). To monitor effects on axon outgrowth, we then performed longer term experiments in which neurons were exposed to BayK for periods up to $24 \mathrm{~h}$ (Fig. 5). In some cases, images were acquired every $2 \mathrm{~h}$ (Fig. 5A) and in others images were acquired at the beginning and end of the imaging period (Fig. 5B-D). Exposure to BayK had no deleterious effects on the neurons which continued to extend processes robustly. In $64 \%$ of neurons $(n=129)$ exposed to BayK for 10-24 h significant differences in process outgrowth and retraction occurred in axons and their branches (Fig. 5E). As shown in Figure 5B, during the $12 \mathrm{~h}$ exposure to BayK the axon grew $140 \mu \mathrm{m}$ whereas both branches (24 and 45 $\mu \mathrm{m}$ in length) completely retracted. In contrast another neuron (Fig. 5C) initially had an axon $400 \mu \mathrm{m}$ long and numerous branches. After exposure to BayK for $20 \mathrm{~h}$, the axon retracted for $88 \mu \mathrm{m}$ back to a branch point whereas two short branches of 18 and $44 \mu \mathrm{m}$ showed dramatic extension of 100 and $148 \mu \mathrm{m}$, respectively. In contrast only $35 \%$ of control neurons $(n=$ 130) showed significant differences in outgrowth and retraction in axons versus branches (Fig. 5D,E). Interestingly, differential rates of process outgrowth in BayK experiments more often involved complete retraction of an axonal process compared with controls in which one or more processes grew more rapidly than another. Because BayK opens L-type channels leading to increased $\mathrm{Ca}^{2+}$ activity, we measured whether overall axon outgrowth was decreased by BayK. This was indeed the case because overall axon outgrowth was reduced to $67 \pm 6 \%$ of control rates $(p<0.001, t$ test $)$. This is consistent with our previous results in which high $\mathrm{Ca}^{2+}$ activity was correlated with reduced axon outgrowth (Tang et al., 2003).

Although BayK reduced overall rates of axon outgrowth, we found that it increased differential growth of axons and their branches in comparison with untreated controls. It was not possible to image $\mathrm{Ca}^{2+}$ transients throughout the long term BayK experiments without compromising the ability of axons to ex-

$\mathrm{B}$
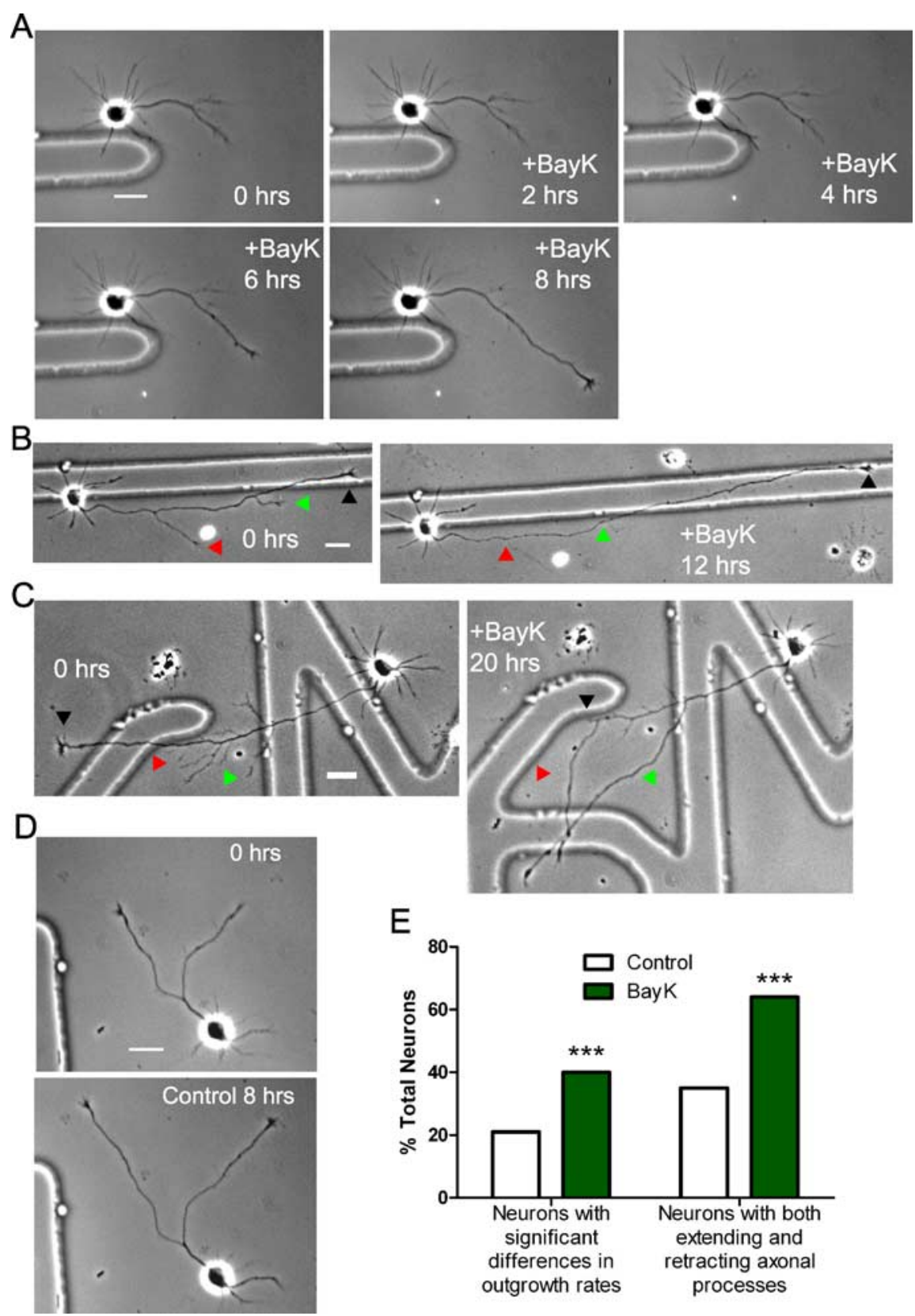

E
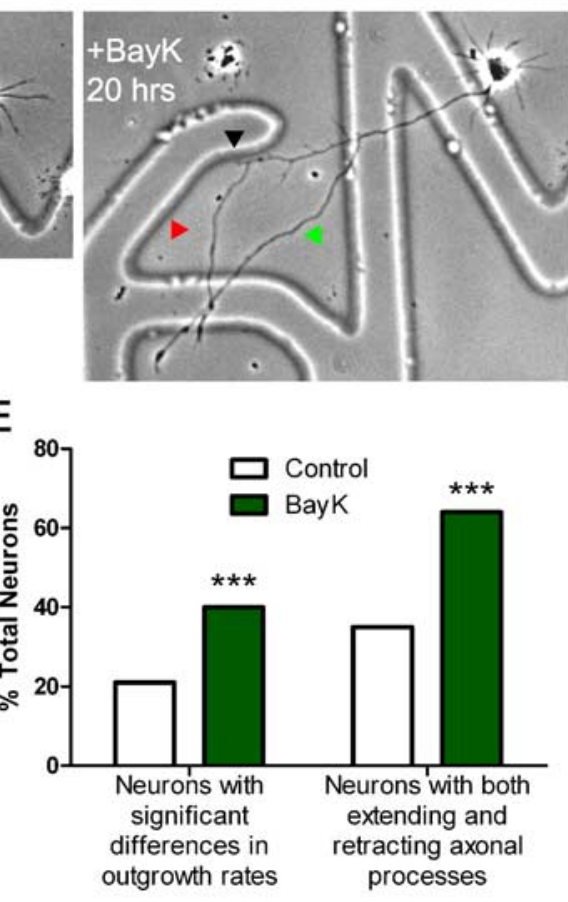

Figure 5. Bath application of BayK 8644 increases differential process outgrowth. $A$, Time-lapse images of a cortical neuron exposed to BayK for $8 \mathrm{~h}$, showing extension of the primary axon and retraction of the branch. $B, C$, Phase images showing the effect of BayK on increasing differential outgrowth between different processes from the same axon. $B$, After $12 \mathrm{~h}$ of exposure to Bayk, the primary axon (black arrowhead) extended whereas the two branches (green and red arrowheads) completely retracted. C, After $20 \mathrm{~h}$ of exposure to BayK the primary axon (black arrowhead) retracted back to the nearest branch point whereas two branches (green and red arrowheads) extended. $\boldsymbol{D}$, An example of a control neuron with only small differences in rates of outgrowth during the imaging session. In contrast to BayK treatment, differences in rates of outgrowth are smaller in control cultures. $\boldsymbol{E}$, Quantifications of the effect of BayK on increasing differential outgrowth. $\boldsymbol{E}$, Left, Bath application of BayK increased the number of neurons with significant differences in outgrowth rates among their axonal processes compared with control $\left({ }^{* * *} p<0.001\right.$, Fisher's exact test, $n \geq 129$ neurons from 5 experiments) (see Materials and Methods). Right, Bath application of BayK significantly increased the number of neurons that had both extending and retracting axonal processes $\left({ }^{* * *} p<0.001\right.$, Fisher's exact test, $n \geq 129$ neurons from 5 experiments). Scale bar, $20 \mu \mathrm{m}$. tend. Therefore to correlate $\mathrm{Ca}^{2+}$ activity evoked by BayK with rates of process outgrowth, we performed $\mathrm{Ca}^{2+}$ imaging during the last 10-15 min of BayK exposure in 16 experiments. In 14 of these neurons, localized differential $\mathrm{Ca}^{2+}$ transients were observed in axons and branches. In the example shown in Figure $6 \mathrm{~A}$, the neuron before the addition of BayK had two major axonal processes, but after $12 \mathrm{~h}$ of BayK exposure, the left process was 

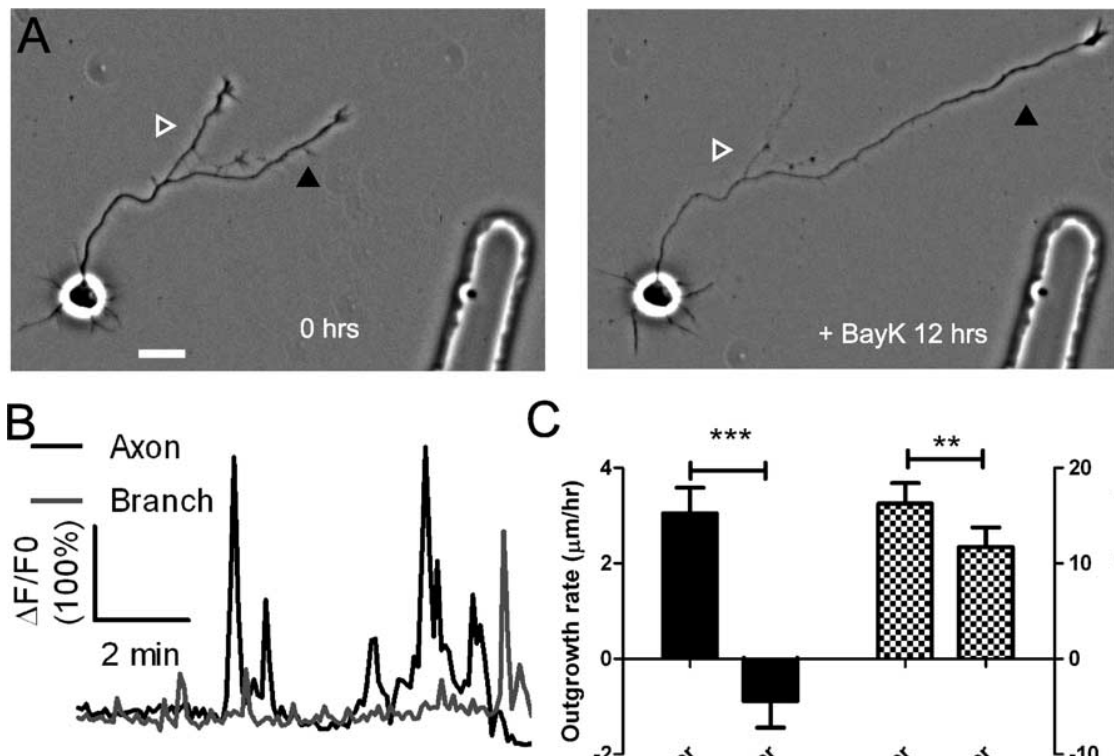

C

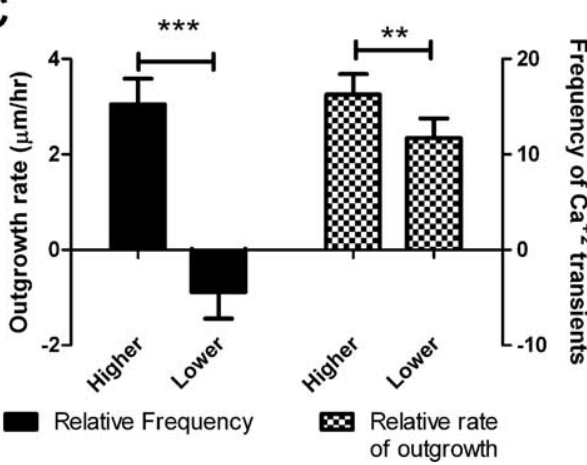

Figure 6. BayK-induced calcium transients persist throughout long term treatments. $\boldsymbol{A}$, Phase images of a cortical neuron treated with BayK for $12 \mathrm{~h}$. At the end of the imaging session the processes on right (black arrowhead) extended, whereas the process on the left retracted (white/gray arrowhead). $\boldsymbol{B}$, After phase imaging, cortical neurons were loaded with Fluo-4 and calcium activity was measured. The extending process from $\boldsymbol{A}$ (black) showed high levels of calcium activity compared with the retracting process (gray). C, Quantifications from 16 experiments imaging calcium activity after long-term BayK treatments revealed correlations between BayK-induced differential calcium activity and outgrowth. C, Left, Comparisons between processes from the same axon showed that processes with higher frequencies of BayK-induced calcium transients had higher rates of outgrowth, whereas processes with lower frequencies retracted ( $n=22$ comparisons; ${ }^{* * *} p<0.001$, paired $t$ test). Right, Processes that extended faster had higher frequencies of calcium transients ( $n=26$ comparisons; ${ }^{* *} p<0.01$, paired $t$ test). Scale bar, $20 \mu \mathrm{m}$.

undergoing retraction, whereas the other process had extended. Higher-frequency $\mathrm{Ca}^{2+}$ transients were found in the extending process than in the retracting process (Fig. $6 \mathrm{~B}$ ). The correlation between higher-frequency $\mathrm{Ca}^{2+}$ transients and increased rates of process outgrowth versus lower-frequency $\mathrm{Ca}^{2+}$ transients associated with retraction or slower outgrowth growth was observed in almost all (13 of 16) of the long-term BayK experiments in which $\mathrm{Ca}^{2+}$ activity was measured (Fig. 6C).

Given our previous results (Tang and Kalil, 2005) showing that local application of netrin- 1 can evoke localized $\mathrm{Ca}^{2+}$ transients in regions of the axon concomitant with promotion of branches from these same regions, we investigated whether differential $\mathrm{Ca}^{2+}$ activity evoked by netrin-1 could also elicit differential outgrowth of axons and branches. In contrast to the BayK experiments, the rapid effects of local netrin-1 application on axon branching made it possible to measure $\mathrm{Ca}^{2+}$ activity simultaneously with axon outgrowth. Of 15 neurons that expressed localized $\mathrm{Ca}^{2+}$ activity in their axons in response to local application of netrin-1, five neurons responded by developing branches de novo in the region of evoked $\mathrm{Ca}^{2+}$ activity. In one example (Fig. $7 A, B$ ) during the 30 min observation period, local application of netrin-1 elicited extension of two branches in the vicinity of the pipette tip. Measurements of $\mathrm{Ca}^{2+}$ activity in this region showed that the highest frequencies occurred in the developing branches. Importantly the branches show the highest rates of outgrowth compared with the distal region of the axon which retracts and shows lower-frequency transients. Measurements of frequencies of $\mathrm{Ca}^{2+}$ transients in 15 neurons in which local application of netrin-1-evoked localized $\mathrm{Ca}^{2+}$ activity revealed that higher frequencies in a branch or an axon were correlated with higher rates of outgrowth concomitant with reduced outgrowth or retraction of another process of the same axon $(6.9 \pm 1.9 \mu \mathrm{m} / \mathrm{h}$ vs $-4.4 \pm$ $1.6 \mu \mathrm{m} / \mathrm{h} ; p<0.0001$, paired $t$ test $)$.

\section{Localized $\mathrm{Ca}^{2+}$ transients evoked by photolysis of caged calcium promote differential outgrowth of axons and their branches}

To determine whether axon outgrowth is promoted when localized $\mathrm{Ca}^{2+}$ transients are experimentally evoked, we sought to restrict $\mathrm{Ca}^{2+}$ activity to defined regions of axonal processes. We therefore used photolysis of caged calcium to evoke repetitive transients in localized regions of axons and their branches. We chose a frequency of $\mathrm{Ca}^{2+}$ transients close to the average transients (40 transients/h) observed previously (Tang and Kalil, 2005) in axonal branches of netrin-1 stimulated neurons undergoing relatively rapid outgrowth. Because the spot exposed to UV light was $\sim 50$ $\mu \mathrm{m}$ in diameter, we were able to restrict photolysis to regions $(12-50 \mu \mathrm{m})$ of axons or branches that typically included the distal region of the process and its growth cone. In the first set of experiments $(n=7)$, we verified that our photolysis procedures were able to evoke localized $\mathrm{Ca}^{2+}$ transients. Neurons with at least one branch were loaded with Fluo-4 and NP-EGTA (caged calcium) and a chosen region of the axon was stimulated at the desired frequency with repetitive pulses of UV light. As shown in Figure 8, $A$ and $B$, each UV stimulation reliably evoked an immediate $\mathrm{Ca}^{2+}$ transient in the region of interest that was on average $106.5 \%$ of baseline $\mathrm{Ca}^{2+}$ levels (Fig. $8 C$ ). Although this was smaller than the average increase in amplitude that we found for spontaneous localized $\mathrm{Ca}^{2+}$ activity (175\% of baseline) this increase was consistent with that evoked by photolysis in previous studies in which a similar increase in amplitude of $\mathrm{Ca}^{2+}$ activity was shown to induce growth cone turning (Tojima et al., 2007) or prevent neurite retraction (Lohmann et al., 2002). To further confirm that photolysis reliably evoked $\mathrm{Ca}^{2+}$ activity we increased the UV light intensity by four times and found that the amplitude of evoked $\mathrm{Ca}^{2+}$ activity was increased to $122 \%$ of baseline (Fig. 8D).

To determine whether evoked $\mathrm{Ca}^{2+}$ activity increased rates of axon outgrowth, we performed photolysis for $30 \mathrm{~min}$ on neurons $(n=21)$ with at least one axon branch and then compared outgrowth of the stimulated and unstimulated axons and their branches. NP-EGTA is a calcium chelator with a low dissociation constant and it has been shown that neurons loaded with NPEGTA do not express spontaneous $\mathrm{Ca}^{2+}$ transients (Gomez et al., 2001). As shown in Figure 8 in the majority of cases (67\%), the stimulated process was favored for growth compared with the unstimulated process (Fig. 8G). This was true whether the stimulated process was an axon or a branch, which were stimulated in equal numbers. As shown in Figure $8 E$, stimulation of the distal region of the primary axon for $30 \mathrm{~min}$ resulted in axon growth of $17.4 \mu \mathrm{m}$, approximately three times the average for unstimulated control neu- 
rons, in contrast to the unstimulated branch which retracted $5.6 \mu \mathrm{m}$. In another example (Fig. $8 F$ ), photolysis was performed on the lower axonal process which extended $\sim 10$ $\mu \mathrm{m}$ whereas the unstimulated process retracted $\sim 15 \mu \mathrm{m}$. Thus, in each case the unstimulated process served as an internal control for potentially harmful effects of photolysis which might be expected to impair neurite outgrowth. In addition, we performed control experiments by exposing axon processes to flashes of UV light in the absence of caged calcium. As expected, exposure to UV light reduced outgrowth of the exposed processes compared with the unexposed processes (Fig. 8G). Taking this negative effect on axon outgrowth into account, the effects of evoked localized $\mathrm{Ca}^{2+}$ activity on increasing axon outgrowth may have been underestimated in our measurements. Together, results from the photolysis induced and spontaneous $\mathrm{Ca}^{2+}$ activity show the same competitive effects on the growth of one axonal process at the expense of another.

\section{Silencing localized $\mathrm{Ca}^{2+}$ transients} reduces differences in growth rates in processes of the same axon

Having shown that localized $\mathrm{Ca}^{2+}$ transients can favor outgrowth of one axonal process over another, we sought to determine whether silencing localized $\mathrm{Ca}^{2+}$ activity would reduce or eliminate disparities in rates of process outgrowth. BayK induces $\mathrm{Ca}^{2+}$ activity through L-type voltage-gated channels. Therefore to silence $\mathrm{Ca}^{2+}$ transients, we used nifedipine, which we previously found to block $\mathrm{Ca}^{2+}$ entry through L-type channels on cortical neurons. As shown in Figure 9A, nifedipine blocked most localized activity and consequently reduced differential outgrowth of axons and their branches (Fig. 9C), an effect which persisted in long term exposure to nifedipine up to $24 \mathrm{~h}$ (Fig. $9 D, E)$. Nifedipine reduced differential process outgrowth while at the same time increasing the overall outgrowth of axons and their branches to $120 \pm 7 \%$ of control rates $(p<0.05, t$ test), consistent with our previous results showing that nifedipine increases rates of cortical axon outgrowth (Tang et al., 2003). As an additional means to reduce $\mathrm{Ca}^{2+}$ transients we applied TTX to neurons exhibiting localized $\mathrm{Ca}^{2+}$ activity. Compared with the frequencies of transients before treatment, which averaged 14.4 per hour, TTX reduced $\mathrm{Ca}^{2+}$ activity to an average of 7.2 per hour (Fig. 9B). Concomitantly, differences in outgrowth rates of axons and their branches were reduced by a factor of 3 compared with untreated control neurons (Fig. 9C).

\section{Discussion}

In the present study, we investigated the role of localized calcium signaling in differential outgrowth of axons and their branches. We found that, in addition to global $\mathrm{Ca}^{2+}$ activity, axons and their branches can express spontaneous $\mathrm{Ca}^{2+}$ transients of different frequencies, which were localized to small regions of axons or restricted to single branches. Measurements of rates of outgrowth showed that higher frequencies of $\mathrm{Ca}^{2+}$ transients were correlated with higher rates of process outgrowth. Surprisingly, we observed that when an axonal process with higher-frequency $\mathrm{Ca}^{2+}$ transients extended relatively rapidly another process of the same axon with lower-frequency $\mathrm{Ca}^{2+}$ transients either stalled or retracted. This suggested a competitive mechanism whereby processes with higher-frequency $\mathrm{Ca}^{2+}$ transients extended at the expense of processes with lower levels of $\mathrm{Ca}^{2+}$ activity. To support these results experimentally we imposed $\mathrm{Ca}^{2+}$ activity pharmacologically with BayK, physiologically with netrin-1 or with localized photolysis of caged calcium. In each case, imposition of repetitive $\mathrm{Ca}^{2+}$ activity in one axonal process led to growth of that process, but stalling or retraction of the unstimulated branch. Conversely, pharmacologically silencing $\mathrm{Ca}^{2+}$ activity reduced the disparities in growth of axons and their branches. Together, these results suggest a competitive frequencydependent mechanism whereby localized $\mathrm{Ca}^{2+}$ activity selec- 

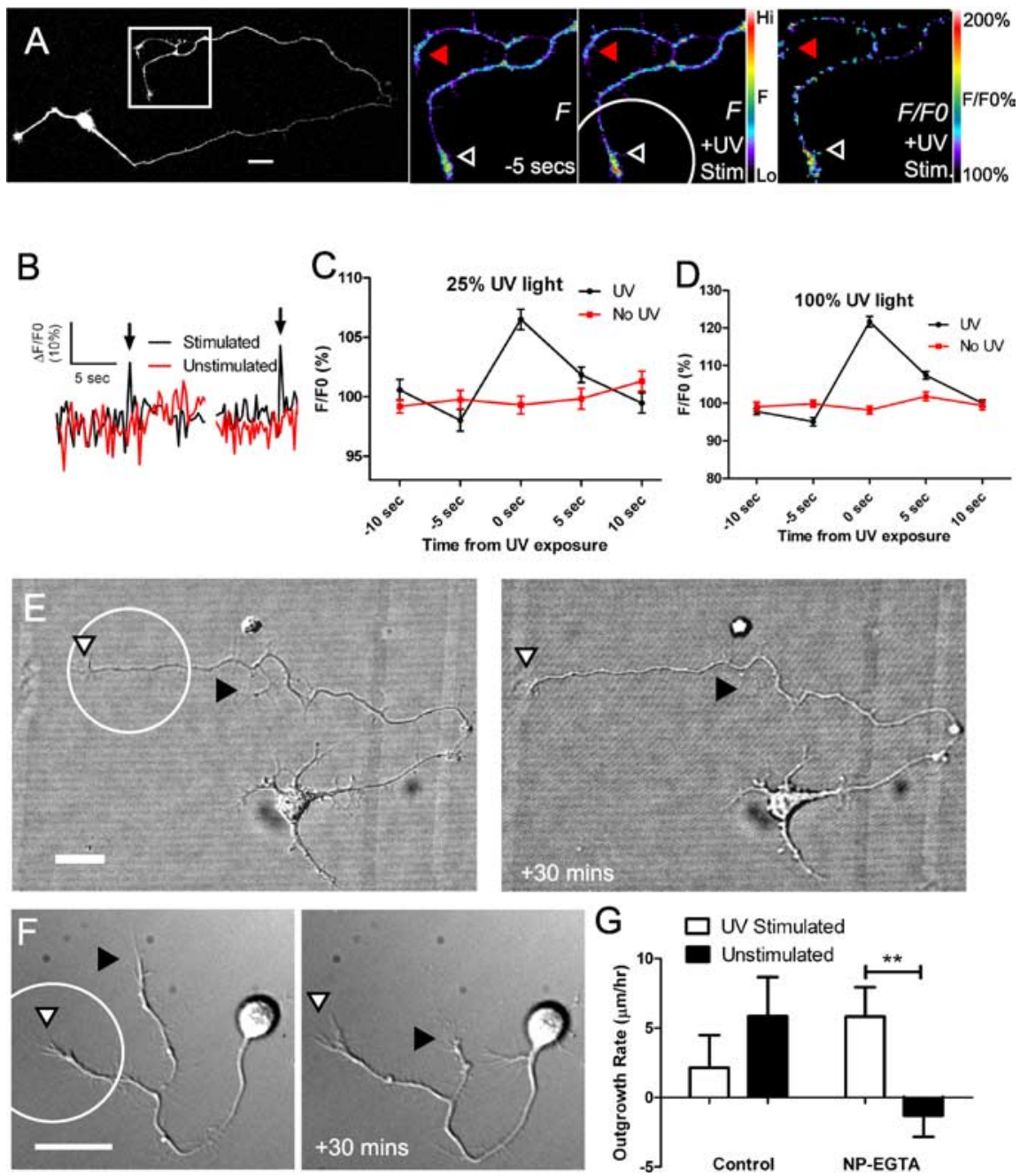

Figure 8. Localized photolysis of caged calcium increases differential outgrowth of stimulated vs unstimulated processes. $\boldsymbol{A}$, Fluorescence image of a cortical neuron loaded with NP-EGTA and Flu0-4. Fluorescence images of calcium activity at rest $(-5 \mathrm{~s})$ and during UV stimulation of the bottom process (+UV Stim; circle indicates the stimulated region) show a localized calcium transient caused by photorelease of caged calcium (pseudocolor scale bar on right). Normalized fluorescence image indicates the change in fluorescence compared with baseline (F/FO + UV Stim; pseudocolor scale on right). Arrowheads indicate unstimulated (red) and stimulated (black) regions of the axon where calcium activity was measured. $\boldsymbol{B}$, Graph of calcium activity over time during the experiment shown in $\boldsymbol{A}$. Exposure to UV light increases calcium activity in the stimulated region (black), but not in the unstimulated process (red). Arrows indicate the time point of UV exposure. C, Quantification of amplitudes of calcium activity evoked by stimulation with UV light filtered to $25 \%$ of maximum intensity in seven experiments. Stimulation increased fluorescence intensity to $106.5 \%$ of baseline in stimulated (black) but not unstimulated (red) processes ( $n \geq 40$ measurements). All outgrowth experiments were performed at this light intensity. $\boldsymbol{D}$, Increasing the intensity of the UV light to $100 \%$ of maximum intensity increased the amplitude of the evoked response to $122 \%$ of baseline in five experiments. $\boldsymbol{E}$, DIC images of a cortical neuron before (left) and after (right) repetitive stimulation in the region indicated by the circle. The primary axon (white arrowhead) extended $17.4 \mu \mathrm{m}$ whereas the branch (black arrowhead) retracted. $\boldsymbol{F}$, Higher-magnification DIC images of a cortical neuron before (left) and after (right) repetitive stimulation. The stimulated process (indicated with a circle) extended $8.5 \mu \mathrm{m}$ whereas the unstimulated process retracted $23 \mu \mathrm{m}$. G, Quantification of outgrowth in stimulated (black bars) versus unstimulated processes $\left({ }^{* *} p<0.01, t\right.$ test). Control neurons were not loaded with NP-EGTA, but were stimulated with UV light $(n=21$ NP-EGTA loaded neurons and 18 control neurons). Scale bars, $20 \mu \mathrm{m}$.

tively promotes growth of one axonal process at the expense of another with $\mathrm{Ca}^{2+}$ activity of lower frequency.

Previous studies from our laboratory (Tang et al., 2003; Tang and Kalil, 2005) have shown that the growth of axons and branches of developing cortical neurons is regulated by levels of calcium activity. For spontaneous activity, we found that highfrequency high-amplitude global $\mathrm{Ca}^{2+}$ transients inhibited axon outgrowth and that silencing L-type voltage-gated $\mathrm{Ca}^{2+}$ channels promoted axon outgrowth (Tang et al., 2003). Although the present study showed that localized $\mathrm{Ca}^{2+}$ transients promoted differential axonal outgrowth, we also found that BayK, which opens L-type channels leading to increased $\mathrm{Ca}^{2+}$ activity, decreased overall axon outgrowth, consistent with our previous findings for global transients, in which rates of axon growth were inversely proportional to levels of calcium activity (Tang et al., 2003). For calcium activity induced by application of netrin-1, we found that the emergence of branches from the axon shaft was promoted by high-frequency localized $\mathrm{Ca}^{2+}$ transients that evoked localized calcium/ calmodulin dependent protein kinase II (CaMKII) and mitogen-activated protein kinase signaling (Tang and Kalil, 2005). The present results are consistent with these findings because the growth of axonal processes was correlated with higherfrequency localized $\mathrm{Ca}^{2+}$ transients. Importantly, however, we show here for the first time that axons and their branches or different branches from the same axon can exhibit spontaneous calcium transients of different frequencies and that the relative differences in these frequencies as opposed to absolute levels of $\mathrm{Ca}^{2+}$ activity were responsible for differential and competitive growth of axonal processes.

\section{Activity-dependent mechanisms govern competitive axon outgrowth}

Previously, spontaneous fluctuations in intracellular calcium at early stages of development have been shown to reflect electrical activity (Cossart et al., 2005; Spitzer, 2006). Previous studies have described a role for activity-dependent mechanisms in the competition among different axons for synaptic territory during neural development (Sengpiel and Kind, 2002; Uesaka et al., 2006). In general, axons with higher levels of electrical activity outcompete axons with lower activity levels. A previous study in the zebrafish visual system showed that electrical activity in one axon relative to neighboring axons is a key determinant for elaborating a terminal arbor (Hua et al., 2005). Silencing electrical activity in single neurons by expressing an inward rectifying potassium channel decreased the size of their terminal axon arbors, and silencing neighboring axons with TTX rescued the arbor size and complexity of the transfected neurons. These results suggest that electrical activity per se confers a competitive advantage to axons with higher levels of electrical activity. Several previous studies support the role of activity in differentiation of CNS neurons. For example, Uesaka et al. (2007) demonstrated in organotypic cocultures of thalamus and cortex that neural activity enhanced the branch dynamics of thalamocortical axons that were biased toward more branch addition and elongation of branches in specific cortical target layers. The importance of electrical activity was also shown in vivo because reducing neuronal 
activity by overexpressing an inwardrectifying $\mathrm{K}^{+}$channel in cortical neurons inhibited their morphological maturation including neurite length and branch number (Cancedda et al. 2007). In addition, eliminating correlated waves of electrical activity during development of the mammalian visual system disrupts pruning of axonal branches and refinement of the retinotopic map (McLaughlin et al., 2003; Chandrasekaran et al., 2005) supporting an instructive role for electrical activity in interaxonal competition.

In contrast, the role of differential $\mathrm{Ca}^{2+}$ activity in regulating competitive outgrowth among different processes of the same axon has been little studied. A previous study of NGF-mediated axon outgrowth demonstrated that local depolarization of axons with $\mathrm{KCl}$ to induce calcium entry resulted in increased outgrowth of those processes at the expense of outgrowth in unstimulated branches and neighboring axons (Singh and Miller, 2005). Furthermore, this competitive outgrowth was dependent on calcium influx through voltage gated channels and downstream activation of CaMKII. Because $\mathrm{Ca}^{2+}$ activity was not measured, it is not known whether the effects on outgrowth were frequency dependent. Thus, our results based on careful measurements of $\mathrm{Ca}^{2+}$ activity support the novel finding that higher frequencies of $\mathrm{Ca}^{2+}$ transients confer a competitive advantage for growth of one branch over another.

\section{Branching of cortical neurons in vitro reflects in vivo branching mechanisms} In vivo, axons and branches projecting to different regions of the nervous system are likely to encounter different types and concentrations of guidance cues such as netrin-1 that evoke different levels of $\mathrm{Ca}^{2+}$ activity leading to competition among different processes of the same axon for growth. This mechanism would permit the primary axon to stall or regress while a branch of the same axon extends toward targets, as has been observed directly in the callosal pathway within cortical slices (Halloran and Kalil, 1994). How might differences in frequencies of $\mathrm{Ca}^{2+}$ transients favor the growth of one process over another? It seems likely that differential activation of calcium frequency-dependent effectors such as CaMKII and calcineurin would occur in each axonal process. Positive effectors such as CaMKI and CaMKII, which have been shown to promote neurite outgrowth (Borodinsky et al., 2003; Wayman et al., 2004, 2006) are preferentially activated in axonal processes with higher frequencies of $\mathrm{Ca}^{2+}$ transients (Tang and Kalil, 2005). CaMKII $\beta$ has been shown to bind directly to actin filaments and increase filopodial dynamics (Fink et al., 2003), consistent with results indicating that direct elevation of $\mathrm{Ca}^{2+}$ activity by photolysis of caged calcium can rapidly evoke protrusion of new filopodia through polymerization of actin filaments (Lau et al., 1999). Different frequencies of $\mathrm{Ca}^{2+}$ transients may target different kinases and phophatases such as CaMKII and calcineurin, respectively, in the cytoplasm to elicit different cellular responses (Tomida et al., 2003). Opposing events such as extension and retraction of axonal processes may be regulated by $\mathrm{Ca}^{2+}$ transients at optimal frequencies (Eshete and Fields, 2001). Interestingly, we found here that for rapid extension of cortical axons and branches the optimal frequencies of both spontaneous and induced localized $\mathrm{Ca}^{2+}$ transients were similar across experiments. Thus, one role for localized $\mathrm{Ca}^{2+}$ signaling may be to confine $\mathrm{Ca}^{2+}$ transients at optimal frequencies to localized regions of the axon. Localized $\mathrm{Ca}^{2+}$ signaling would thereby regulate extension and retraction of axonal processes in response to environmental growth and guidance cues such as netrin-1, which we have shown previously to evoke high-frequency $\mathrm{Ca}^{2+}$ transients and induce axon branching (Tang and Kalil, 2005). At present, the signaling mechanisms whereby the growth of one axonal process is coordinated with the retraction of another are not known. However, we show here for the first time that localized $\mathrm{Ca}^{2+}$ transients are required for frequency dependent competitive growth of axons and their branches.

\section{References}

Augustine GJ, Santamaria F, Tanaka K (2003) Local calcium signaling in neurons. Neuron 40:331-346. 
Bastmeyer M, O'Leary DD (1996) Dynamics of target recognition by interstitial axon branching along developing cortical axons. J Neurosci 16:1450-1459.

Borodinsky LN, O’Leary D, Neale JH, Vicini S, Coso OA, Fiszman ML (2003) GABA-induced neurite outgrowth of cerebellar granule cells is mediated by $\mathrm{GABA}(\mathrm{A})$ receptor activation, calcium influx and CaMKII and erk1/2 pathways. J Neurochem 84:1411-1420.

Cancedda L, Fiumelli H, Chen K, Poo MM (2007) Excitatory GABA action is essential for morphological maturation of cortical neurons in vivo. J Neurosci 27:5224-5235.

Chandrasekaran AR, Plas DT, Gonzalez E, Crair MC (2005) Evidence for an instructive role of retinal activity in retinotopic map refinement in the superior colliculus of the mouse. J Neurosci 25:6929-6938.

Cossart R, Ikegaya Y, Yuste R (2005) Calcium imaging of cortical networks dynamics. Cell Calcium 37:451-457.

Dent EW, Kalil K (2003) Dynamic imaging of neuronal cytoskeleton. Methods Enzymol 361:390-407.

Dent EW, Barnes AM, Tang F, Kalil K (2004) Netrin-1 and semaphorin 3A promote or inhibit cortical axon branching, respectively, by reorganization of the cytoskeleton. J Neurosci 24:3002-3012.

Eshete F, Fields RD (2001) Spike frequency decoding and autonomous activation of $\mathrm{Ca}^{2+}$-calmodulin-dependent protein kinase II in dorsal root ganglion neurons. J Neurosci 21:6694-6705.

Fink CC, Bayer KU, Myers JW, Ferrell Jr JE, Schulman H, Meyer T (2003) Selective regulation of neurite extension and synapse formation by the beta but not the alpha isoform of CaMKII. Neuron 39:283-297.

Goldberg JL, Espinosa JS, Xu Y, Davidson N, Kovacs GT, Barres BA (2002) Retinal ganglion cells do not extend axons by default: promotion by neurotrophic signaling and electrical activity. Neuron 33:689-702.

Gomez TM, Spitzer NC (1999) In vivo regulation of axon extension and pathfinding by growth-cone calcium transients. Nature 397:350-355.

Gomez TM, Zheng JQ (2006) The molecular basis for calcium-dependent axon pathfinding. Nat Rev Neurosci 7:115-125.

Gomez TM, Robles E, Poo M, Spitzer NC (2001) Filopodial calcium transients promote substrate-dependent growth cone turning. Science 291:1983-1987.

Halloran MC, Kalil K (1994) Dynamic behaviors of growth cones extending in the corpus callosum of living cortical brain slices observed with video microscopy. J Neurosci 14:2161-2177.

Henley J, Poo MM (2004) Guiding neuronal growth cones using $\mathrm{Ca}^{2+}$ signals. Trends Cell Biol 14:320-330.

Henley JR, Huang KH, Wang D, Poo MM (2004) Calcium mediates bidirectional growth cone turning induced by myelin-associated glycoprotein. Neuron 44:909-916.

Hua JY, Smear MC, Baier H, Smith SJ (2005) Regulation of axon growth in vivo by activity-based competition. Nature 434:1022-1026.

Jacques-Fricke BT, Seow Y, Gottlieb PA, Sachs F, Gomez TM (2006) $\mathrm{Ca}^{2+}$ influx through mechanosensitive channels inhibits neurite outgrowth in opposition to other influx pathways and release from intracellular stores. J Neurosci 26:5656-5664.

Lau PM, Zucker RS, Bentley D (1999) Induction of filopodia by direct local elevation of intracellular calcium ion concentration. J Cell Biol 145:1265-1275.
Lohmann C, Myhr KL, Wong RO (2002) Transmitter-evoked local calcium release stabilizes developing dendrites. Nature 418:177-181.

Luo L, O'Leary DD (2005) Axon retraction and degeneration in development and disease. Annu Rev Neurosci 28:127-156.

McLaughlin T, Torborg CL, Feller MB, O’Leary DD (2003) Retinotopic map refinement requires spontaneous retinal waves during a brief critical period of development. Neuron 40:1147-1160.

Meyer MP, Smith SJ (2006) Evidence from in vivo imaging that synaptogenesis guides the growth and branching of axonal arbors by two distinct mechanisms. J Neurosci 26:3604-3614.

Nishiyama M, Hoshino A, Tsai L, Henley JR, Goshima Y, Tessier-Lavigne M, Poo MM, Hong K (2003) Cyclic AMP/GMP-dependent modulation of $\mathrm{Ca}^{2+}$ channels sets the polarity of nerve growth-cone turning. Nature 423:990-995.

Portera-Cailliau C, Weimer RM, De Paola V, Caroni P, Svoboda K (2005) Diverse modes of axon elaboration in the developing neocortex. PLoS Biol 3:e272.

Ruthel G, Hollenbeck PJ (2000) Growth cones are not required for initial establishment of polarity or differential axon branch growth in cultured hippocampal neurons. J Neurosci 20:2266-2274.

Sengpiel F, Kind PC (2002) The role of activity in development of the visual system. Curr Biol 12:R818-R826.

Singh KK, Miller FD (2005) Activity regulates positive and negative neurotrophin-derived signals to determine axon competition. Neuron 45:837-845.

Spitzer NC (2006) Electrical activity in early neuronal development. Nature 444:707-712.

Tang F, Kalil K (2005) Netrin-1 induces axon branching in developing cortical neurons by frequency-dependent calcium signaling pathways. J Neurosci 25:6702-6715.

Tang F, Dent EW, Kalil K (2003) Spontaneous calcium transients in developing cortical neurons regulate axon outgrowth. J Neurosci 23:927-936.

Tojima T, Akiyama H, Itofusa R, Li Y, Katayama H, Miyawaki A, Kamiguchi $\mathrm{H}$ (2007) Attractive axon guidance involves asymmetric membrane transport and exocytosis in the growth cone. Nat Neurosci 10:58-66.

Tomida T, Hirose K, Takizawa A, Shibasaki F, Iino M (2003) NFAT functions as a working memory of $\mathrm{Ca}^{2+}$ signals in decoding $\mathrm{Ca}^{2+}$ oscillation. EMBO J 22:3825-3832.

Uesaka N, Ruthazer ES, Yamamoto N (2006) The role of neural activity in cortical axon branching. Neuroscientist 12:102-106.

Uesaka N, Hayano Y, Yamada A, Yamamoto N (2007) Interplay between laminar specificity and activity-dependent mechanisms of thalamocortical axon branching. J Neurosci 27:5215-5223.

Wayman GA, Kaech S, Grant WF, Davare M, Impey S, Tokumitsu H, Nozaki N, Banker G, Soderling TR (2004) Regulation of axonal extension and growth cone motility by calmodulin-dependent protein kinase I. J Neurosci 24:3786-3794.

Wayman GA, Impey S, Marks D, Saneyoshi T, Grant WF, Derkach V, Soderling TR (2006) Activity-dependent dendritic arborization mediated by CaM-kinase I activation and enhanced CREB-dependent transcription of Wnt-2. Neuron 50:897-909.

Wen Z, Guirland C, Ming GL, Zheng JQ (2004) A CaMKII/calcineurin switch controls the direction of $\mathrm{Ca}^{2+}$-dependent growth cone guidance. Neuron 43:835-846. 